\title{
RESEARCH ON METHODS OF HIGH COHERENT TARGET EXTRACTION IN URBAN AREA BASED ON PSINSAR TECHNOLOGY
}

\author{
Naiyi Li, Jicang Wu \\ College of Surveying and Geo-Informatics, Tongji University, Shanghai, 1633323@tongji.edu.cn
}

Commission III, WG III/3

KEY WORDS : PS points extraction, Dispersion of intensity, Amplitude threshold, Phase analysis, Ground deformation

\begin{abstract}
:
PSInSAR technology has been widely applied in ground deformation monitoring. Accurate identification of Persistent Scatterers (PS) is key to the success of PSInSAR data processing. In this paper, the theoretic models and specific algorithms of PS point extraction methods are summarized and the characteristics and applicable conditions of each method, such as Coherence Coefficient Threshold method, Amplitude Threshold method, Dispersion of Amplitude method, Dispersion of Intensity method, are analyzed. Based on the merits and demerits of different methods, an improved method for PS point extraction in urban area is proposed, that uses simultaneously backscattering characteristic, amplitude and phase stability to find PS point in all pixels. Shanghai city is chosen as an example area for checking the improvements of the new method. The results show that the PS points extracted by the new method have high quality, high stability and meet the strong scattering characteristics. Based on these high quality PS points, the deformation rate along the line-of-sight (LOS) in the central urban area of Shanghai is obtained by using 35 COSMO-SkyMed X-band SAR images acquired from 2008 to 2010 and it varies from $-14.6 \mathrm{~mm} / \mathrm{year}$ to $4.9 \mathrm{~mm} / \mathrm{year}$. There is a large sedimentation funnel in the cross boundary of Hongkou and Yangpu district with a maximum sedimentation rate of more than $14 \mathrm{~mm}$ per year. The obtained ground subsidence rates are also compared with the result of spirit leveling and show good consistent. Our new method for PS point extraction is more reasonable, and can improve the accuracy of the obtained deformation results.
\end{abstract}

\section{INTRODUCTION}

Ground subsidence has became an universal problem that has disturbed social and economic development (Ferretti et al.,2001;2011), timely monitoring of urban ground subsidence is of great significance for the safety assessment of major urban infrastructure and disaster prevention and reduction. In recent years, Persistent Scatterer Interferometric Synthetic Aperture Radar (PSInSAR) technology has been widely applied in ground subsidence and crustal deformation monitoring (Ferretti et al.,2011;Hooper et al.,2008). The research object of PSInSAR is the Persistent Scatterer (PS), so accurate identification of Persistent Scatterers is key to the success of PSInSAR data processing (Hooper,2007).

In the urban area, PS points generally correspond to artificial objects, such as buildings, bridges, road boundaries, embankments, and artificial corner reflectors. PS points extracted by conventional method in city are very dense (Fan et al.,2016). However, due to the high-rising building in city, SAR images overlap and multi-path effect are serious, the selected PS points are unreliable. Selecting the high quality of PS points to improve the accuracy and reliability of PSInSAR deformation results has important significance. The main methods of PS point extraction are Coherence Coefficient Threshold method, Amplitude Threshold method, Dispersion of Amplitude method, etc (Liu et al.,2009;Zhang et al.,2009). In 2015, Gao deduced the relationship between the intensity deviation index and the phase standard deviation, and proposed the Dispersion of Intensity method $(\mathrm{Gao}, 2015)$. According to the phase analysis, Hooper (2007) has proposed an algorithm for PS point detection based on interferometric phase spatial correlation analysis.
Firstly, the principle and algorithm model of several common PS point selection methods are summarized. Shanghai city is chosen as an example area, and we use different methods to select PS points and evaluate the accuracy and stability of extracted PS points. Secondly, we propose an improved method based on the combination of amplitude threshold method, dispersion of amplitude method and phase analysis method, and verifies its accuracy and rationality. Finally, PS points extracted by the improved approach are used to calculate deformation rate in the downtown of Shanghai, the results are compared with other methods and spirit leveling results. The accuracy and reliability of the improved method are verified.

\section{METHDOLOGY}

\subsection{Coherence Coefficient Threshold Method}

The coherence coefficient threshold method is the simplest and most direct method of PS detection (Zhang et al.,2009). Because of the stable back scatter characteristics of point target in time series, they can maintain a high coherence in a long time (Wu et al.,2015). Therefore, PS points can be identified based on the coherence coefficient of pixels.

The basic idea of the coherence coefficient threshold method is as follows: for a pixel, a certain size moving window can be selected, the value of the mean coherence factor is estimated by the complex number of pixels in a moving window. After calculated coherence factor in this window, the window is moved and to repeat the calculation. In general, the higher correlation coefficient, the lower the noise of interference phase, means the higher the signal to noise ratio (SNR). Therefore, the threshold is set (Wu et al.,2016). Then all pixels whose mean coherence is larger than the threshold will be chosen as PS point. 


\subsection{Amplitude Threshold Method}

The amplitude threshold method is realized by using the strong reflection characteristic of the pixel. The strong reflection characteristic of the pixel means the high energy of echo signal, the amplitude values are significant, which be presented as white spot on amplitude images (Nie et al.,2012; Liu et al.,2009) In N SAR images which cover the same area, the amplitude of the pixels are analyzed after radiation calibration, and the appropriate threshold are set. Then the pixels whose the amplitude is greater than the threshold will be selected as PS point (Liu ,2009).

\subsection{Dispersion of Amplitude Method}

The high coherence target not only requires high spatial correlation, but also needs to maintain phase stability in time domain. Therefore, we choose the phase standard deviation in time domain to measure as measurement index (Fan et al.,2016). Since the interferometric phase of PSInSAR is influenced by the factors such as baselines and atmospheric changes, so the change of standard deviation is too large to judge. For that, Ferretti et al. (2001) put forward the Dispersion of Amplitude method (DA). This method uses the amplitude stability of the pixel to replace the phase noise level estimation. Only in the case of enough images (more than 30), can correctly estimate the statistical properties of amplitude. The stable scatterer are obtained by analyzing the amplitude dispersion of each pixel in the time series. The theoretical basis of this method is that the noise level of the phase is measured by the amplitude dispersion index of the pixel, thus the stable PS point in the time domain is selected (Nie et al.,2012).

\subsection{Dispersion of Intensity Method}

The dispersion of amplitude method requires radiation calibration, which is relatively complex and inefficient. For this reason, Gao (2015) put forward dispersion of intensity method, that uses intensity dispersion index instead of amplitude dispersion index, the phase standard deviation is measured by intensity deviation index, and deduced a relationship between the intensity deviation index and the phase standard deviation. Like dispersion of amplitude method, the dispersion of intensity method first calculates average time-series intensity (i.e., the square of the amplitude) of each pixel, and the standard deviation of intensity, then deduces the relationship between phase standard deviation and intensity dispersion index (Gao,2015). When the standard deviation of noise is less than threshold (generally set to 0.47 ), the point can be selected as PS point.

\subsection{Phase Analysis Method}

Based on the analysis of phase stability, Hooper et al. proposed an algorithm for PS detection by interference phase spatial correlation analysis (Hooper,2006;2007). This method realizes the recognition of point targets by a series of filtering and iterative operations.

First, remove most of the topographic phase, the wrapped phase of the $x$ th pixel in the $i$ th flattened and topographically corrected interferograms $\phi_{\mathrm{int}, x, i}$ can be written as follows:

$$
\phi_{\mathrm{int}, x, i}=W\left\{\varphi_{\text {def }, x, i}+\varphi_{a t m, x, i}+\Delta \varphi_{o r b, x, i}+\Delta \varphi_{\theta, x, i}+\varphi_{n, x, i}\right\}
$$

where, $W\{$.$\} means wrapping operator, \varphi_{\text {def }, x, i}$ is the phase change caused by the movement of the pixel in the satellite lineof sight (LOS) direction during the time period of images acquired, $\varphi_{a t m, x, i}$ is the atmospheric phase changed between passes, $\Delta \varphi_{o r b, x, i}$ means the residual phase caused by orbit inaccuracies, $\Delta \varphi_{\theta, x, i}$ is the residual topographic phase due to error in the DEM (Liu et al.,2009), and $\varphi_{n, x, i}$ means the phase caused by various noises. In which, $\varphi_{d e f, x, i}, \varphi_{a t m, x, i}$ and $\Delta \varphi_{o r b, x, i}$ are spatially correlated over distances of a specified length, and they are low frequency signals (Fan et al.,2016), which can be separated from the original phase by low-pass filter. Then the low-frequency phase component $\bar{\varphi}_{x, i}$ is obtained. Subtracting the $\bar{\varphi}_{x, i}$ from the interference phase and rewrapping gives:

$$
W\left\{\phi_{\mathrm{int}, x, i}-\bar{\varphi}_{x, i}\right\}=W\left\{\Delta \varphi_{\theta, x, i}^{n c}+\varphi_{n, x, i}^{n c}+\delta_{x, i}\right\}
$$

Where, $\Delta \varphi_{n, x, i}^{n c}$ denotes spatial non-correlation phase part, $\varphi_{\theta, x, i}^{n c}$ means spatial non-correlation phase caused by incident angle error, and $\delta_{x, i}$ means the sum of spatial non-correlation phase caused by deformation, atmospheric delay, and orbit error. Hooper (2008) has deduced the relationship between the phase change of incident angle error $\Delta \varphi_{\theta}$ and the look angle $\theta$ :

$$
\Delta \varphi_{\theta} \approx \frac{4 \pi}{\lambda} B \cos (\theta-\omega) \Delta \theta=\frac{4 \pi}{\lambda} B_{\perp}(\theta) \Delta \theta
$$

where, $\omega$ is the angle between the baseline vector and the horizontal, and $B_{\perp}(\theta)$ is the perpendicular component of the baseline. Accordingly, the $\mathrm{Eq}(2)$ can be rewritten that:

$$
W\left\{\phi_{\mathrm{int}, x, i}-\bar{\varphi}_{x, i}\right\}=W\left\{\frac{4 \pi}{\lambda} B_{\perp, x, i} \Delta \theta_{x}^{n c}+\varphi_{n, x, i}^{n c}+\delta_{x, i}\right\}
$$

where $\Delta \theta_{x}^{n c}$ can be estimated by the least squares method, noted as $\Delta \hat{\theta}_{x}^{n c}$, then phase estimation $\Delta \hat{\varphi}_{\theta, x, i}^{n c}$ can be calculated $(\operatorname{Eq}(3))$. Subtracting $\Delta \hat{\varphi}_{\theta, x, i}^{n c}$ from $\mathrm{Eq}(2)$ leaves:

$$
W\left\{\phi_{\mathrm{int}, x, i}-\bar{\varphi}_{x, i}-\Delta \hat{\varphi}_{\theta, x, i}^{n c}\right\}=W\left\{\varphi_{n, x, i}^{n c}+\delta_{x, i}\right\}
$$

From the above equation, a measure of the phase stability based on the temporal coherence of pixel $x$ can be obtained (Hooer,2008):

$$
\gamma_{x}=\frac{1}{N}\left|\sum_{i=1}^{N} \exp \left\{\sqrt{-1}\left(\phi_{\text {int }, x, i}-\bar{\varphi}_{x, i}-\Delta \hat{\varphi}_{\theta, x, i}^{n c}\right)\right\}\right|
$$

where, $\gamma_{x}$ represents a measure of the phase stability of the pixel, and it can reflect the phase noise level of each pixel and present the stability of the target in time. 
By binning and normalizing the values of $\gamma_{x}$ we can estimate the probability density $P\left(\gamma_{x}\right)$. So the PS probability can be written as (Hooper,2006):

$$
P\left(\gamma_{x}\right)=\alpha p_{p s}\left(\gamma_{x}\right)+(1-\alpha) p_{R}\left(\gamma_{x}\right)
$$

Giving suitable $\alpha$, the $\operatorname{Eq}(7)$ is workable. So for pixel $\mathrm{x}$, the probability being a PS is:

$$
P(x \in P S)=1-\frac{(1-\alpha) p_{R}\left(\gamma_{x}\right)}{p\left(\gamma_{x}\right)}
$$

Using the probability and the coherence in time-domain, we can extract reliable PS points from the PS candidates. In general, PS candidates are selected by the amplitude or intensity dispersion method, and then the PS point is selected by the phase analysis method iteratively.

\subsection{Improved PS extraction method}

The single threshold method can effectively extract PS point, but each method only considers a single feature of PS point, e.g. coherence coefficient threshold method only considers the impact of the neighborhood's PS point on the point signal; the dispersion of amplitude method only considers high SNR characteristics of PS candidates. Therefore the single threshold method can easily lead to misjudgement or omission (Nie et al.,2012).

In the urban area, the terrain is relatively flat, the buildings are dense, there are green belts and water areas, the surface cover types are more, and the situation is complex. In addition, the urban area construction frequency is relatively high, the ground object changes relatively large. It can be seen that if a single method is used to detect PS points, it is easy to cause misjudgements.

Based on the above analysis, considering the permanent scatterer with phase and amplitude stability, under the condition of surface coverage characteristics in view of the urban area, an improved PS extraction method is tried. The amplitude threshold method can extract the pixel with strong backscattering characteristics (see Section 1.2.1), which can be used as the primary method to obtain the subset of PS candidates $\{P S C\}_{1}$. The dispersion of intensity method can eliminate the unstable points in the time domain (see Section 1.2.3), then the subset of PS candidates $\{P S C\}_{2}$ can be obtained. In order to ensure that the obtained PS points have strong reflection characteristics and stability, the intersection of $\{P S C\}_{1}$ and $\{P S C\}_{2}$ is obtained, and PS preliminary candidate subset $\{P S C\}$ is determined. After the preliminary selection, the phase stability of each PS candidate in $\{P S C\}$ is analyzed by the method of phase spatial correlation analysis, and according to the residual phase change of time coherence measure for PS candidate selection, eliminate the PS candidates with great phase noise, eventually get effective PS points.

\section{EXPERIMENT AND ANALYSIS}

\subsection{Study Area and Data Resource}

In order to verify the effectiveness and reliability of the improved PS detection method proposed in this paper, we use 35 scenes of high-resolution COSMO-SkyMed ascending images covering the urban area of Shanghai as data sources. Time span of the image data from December 10, 2008 to November 6, 2010, and the all radar side angles are $40^{\circ}$. SAR interested image cover area is about $9.7 \mathrm{~km} * 11.2 \mathrm{~km}$, center longitude is east $121^{\circ} 30^{\prime}$ and latitude is north $31^{\circ} 17^{\prime}$. This image include Huangpu district (the original Zhabei), Hongkou district, Yangpu district and Pudong area. There are five subway lines passing this area, i.e.3,4,8,10,12 (Fig 1).

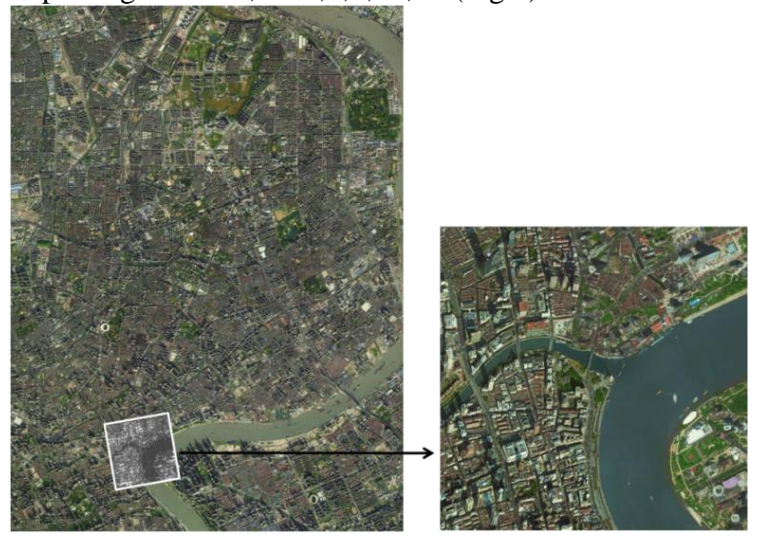

Figure 1. The satellite image of interested area (The picture from Google map)

Considering the effects of time, optimal distribution of spatial baseline, Doppler center frequency and water vapor, the image of 04 March 2009 is selected as the master image and 34 singlelook interferograms are formed. Figure 2 shows the spatial and temporal baseline distribution of the interferograms. The vertical baseline ranges from $21.48 \mathrm{~m}$ to $942.31 \mathrm{~m}$ and the time baseline from 8 days to 368 days.

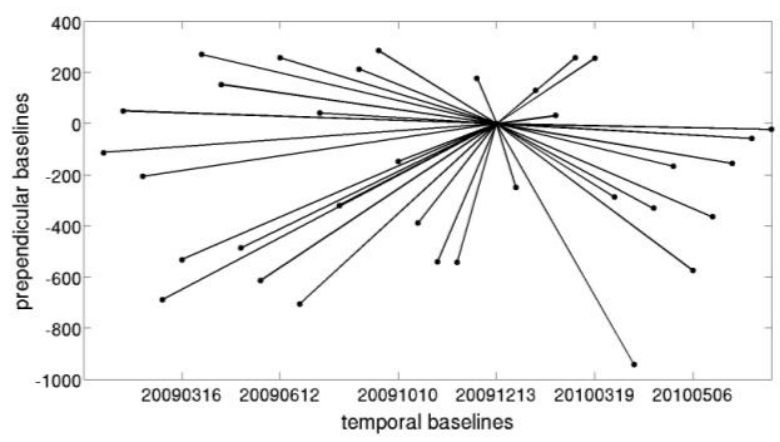

Figure 2. Temporal and spatial baseline

In order to compare the effect of different PS selection methods and improve the experimental efficiency, we select the intersection area of Suzhou river and Huangpu river as the test area of PS point detection (Fig 1). The test area is about $1.94 \mathrm{~km} * 2.25 \mathrm{~km}$. Its northern part belongs to Hongkou district, the south belongs to Huangpu district and the southeast belongs to Pudong area. There are many buildings in the test area, and including water and a small amount of green area. 


\subsection{PS Candidate Selection Results Analysis}

3.2.1 Accuracy and Reliability Analysis: To compare the stability and reliability of each PS method, six different PS point extraction methods are used respectively. These six methods are the Coherence Coefficient Threshold Method, Amplitude Threshold Method, Dispersion of Amplitude Method, Dispersion of Intensity Method, Phase Analysis Method, and our Improved PS Extraction Method.

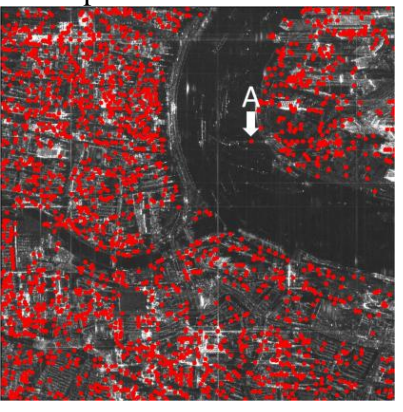

a

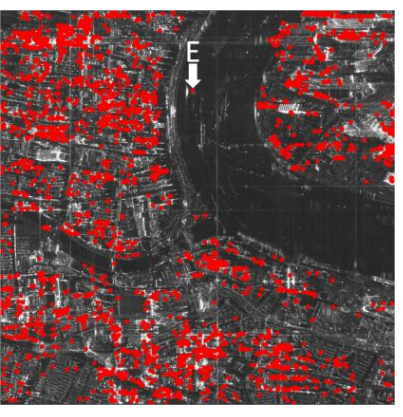

c

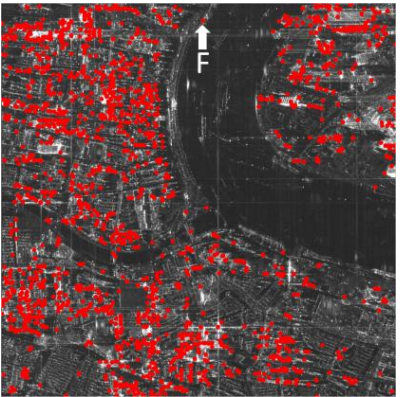

e

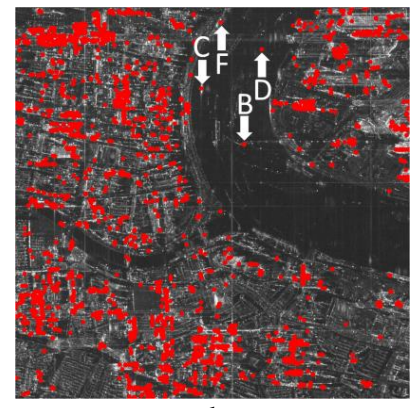

b
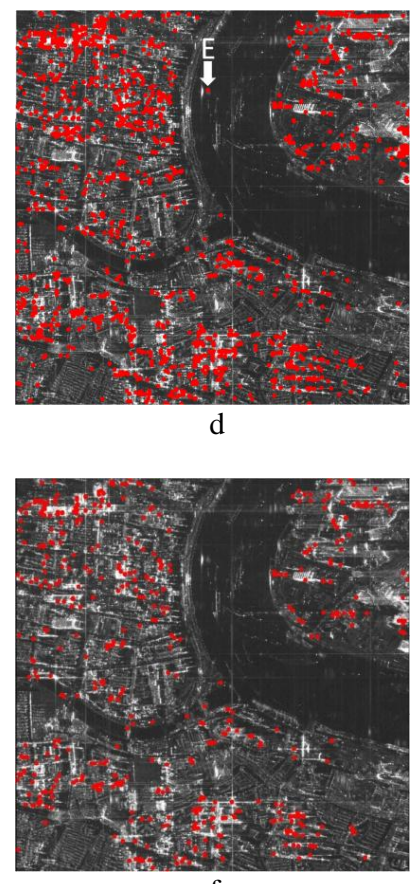

f
Figure 3. The results of six PS selection methods

(a. Coherence coefficient threshold method b. Amplitude threshold method c. Dispersion of Amplitude method d.

Dispersion of Intensity method e. Phase analysis method $\mathrm{f}$. Improved PS point detection method)

The histogram of coherence coefficient of the selected PS points by each method are show in Fig 4. The coherence of this region is low, which are mostly between 0.35 and 0.7. See Figure 4, the coherence value of the selected PS points by the correlation coefficient threshold method, phase analysis method and improved method are greater than 0.45 , and the number of PS points less than 0.45 is 0 . Compared with other methods, the improved method gets higher coherence value of PS point. The result shows that the coherence of PS points obtained by the improved method is higher.

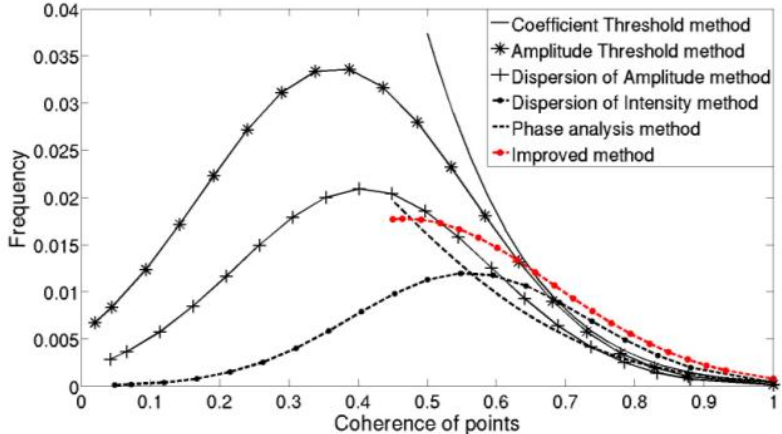

Figure 4. Coherence histogram of PS points

For analysis of the merits and demerits of each method, we choose 6 characteristic points in Figure 3, points A, B, C, D, E and $\mathrm{F}$. The amplitude dispersion, the mean amplitude, amplitude standard deviation, intensity dispersion, and coherence coefficient of characteristics points are listed in Table 1.

\begin{tabular}{cccccc}
\hline ID & $\begin{array}{c}\text { amplitude } \\
\text { dispersion }\end{array}$ & $\begin{array}{c}\text { mean } \\
\text { amplitude }\end{array}$ & $\begin{array}{c}\text { amplitude } \\
\text { standard } \\
\text { deviation }\end{array}$ & $\begin{array}{c}\text { intensity } \\
\text { dispersion }\end{array}$ & $\begin{array}{c}\text { coherence } \\
\text { coefficien } \\
\mathrm{t}\end{array}$ \\
\hline A & 0.37 & 55.41 & 20.53 & 0.58 & 0.57 \\
B & 0.56 & 424.62 & 233.2 & 0.59 & 0.2 \\
C & 0.31 & 169.89 & 50.23 & 0.49 & 0.27 \\
D & 0.52 & 232.26 & 116.15 & 0.59 & 0.2 \\
E & 0.24 & 26.02 & 6.36 & 0.46 & 0.31 \\
F & 0.7 & 325.74 & 228.02 & 0.85 & 0.15 \\
\hline
\end{tabular}

Table 1. The attribute values of PS point

From table 1, it can be seen that the mean amplitude of points B, $\mathrm{C}, \mathrm{D}$ and $\mathrm{F}$ are relatively high, indicating that the corresponding ground object has a higher backscatter. The mean amplitude of points $\mathrm{A}$ and $\mathrm{E}$ are low, mean the radar echo signal is weak, indicating that points $\mathrm{A}$ and $\mathrm{E}$ presents the characteristics of water reflection, they are in the Huangpu river.

Figure 3a shows the distribution of PS points by the correlation coefficient threshold method. It can be seen that the distribution density of the selected PS points is influenced by the calculation of moving windows. Point A is in the Huangpu river, and from Table 1, we can see that the coherent coefficient and amplitude dispersion of this point are higher, but the mean amplitude is small. Although the point has strong correlation, but it does not have strong backscattering characteristics and stability. So point A is invalid PS point. So the correlation coefficient threshold method can cause misjudgment.

Figure $3 \mathrm{~b}$ shows the distribution of PS points by the amplitude threshold method. It can be seen that the points obtained by this method are almost all in the high light of the intensity diagram, which is the ground object with strong backscattering characteristics. According to the actual ground object, point targets are mostly in buildings. Compared with figure 3a, we can find that both points $\mathrm{A}$ and $\mathrm{E}$ with poor backscattering are eliminated. In addition, the mean amplitude of the points B, C, $\mathrm{D}$ and $\mathrm{F}$ are relatively higher (see Table 1 ), these points show the strong backscattering characteristics. However, the amplitude dispersion and intensity dispersion of these 4 points are large, illustrate these points are unstable in time series. Probably, these points are vessels parking over a long period of time in the river, or some new buildings which are built during 
the acquisition of images, so these points are invalid PS points. It can be seen that the amplitude threshold method only considers the scattering characteristic of the pixel, and ignored its stability, thus causes misjudgment. This method has to be improved for considering the stability of PS point.

Figure 3c shows the distribution of PS points by the dispersion of amplitude method. According to the actual objects, the PS points are mostly at the man-made buildings, indicating that the method can extract effective point targets. Compared with figure $3 \mathrm{a}$ and $3 \mathrm{~b}$, the unstable points $\mathrm{A} 、 \mathrm{~B} 、 \mathrm{C} 、 \mathrm{D} 、 \mathrm{~F}$ are removed in figure $3 \mathrm{c}$. In addition, the actual ground object corresponding to point $\mathrm{E}$ is in Huangpu river. According the mean and dispersion of amplitude in Table 1, the mean amplitude of point $\mathrm{E}$ is far less than other points. It is obvious that the scattering strength of $\mathrm{E}$ is always at a lower level. So point $\mathrm{E}$ does not meet the target scattering characteristics, is not a valid target. However, the amplitude dispersion of point $E$ is small, it shows that point $\mathrm{E}$ is stable in time series. Dispersion of amplitude method only consider amplitude dispersion as choosing index, so point $\mathrm{E}$ is misjudged as PS point. This method does not take into account the mean amplitude of the target. For objects with small amplitude or small changes (e.g. waters), but the amplitude dispersion index is small, it is easy to misjudge..

Fig 3d shows the distribution of PS points by dispersion of intensity method. The result of this method is basically the same as that obtained by the dispersion of amplitude method, which eliminates the unstable points $\mathrm{A}, \mathrm{B}, \mathrm{C}, \mathrm{D}$ and $\mathrm{F}$, and also misjudge the point $\mathrm{E}$. Different from the dispersion of amplitude method, this method does not need to conduct radiation correction, and the coherence of the PS point obtained is higher than the dispersion of amplitude method (Fig 4). It can be seen that the calculation process of the dispersion of intensity method is relatively simple, and the quality of PS points result is higher. Therefore, dispersion of amplitude method can be replaced by dispersion of intensity method.

Figure $3 \mathrm{e}$ shows the distribution of PS points by phase analysis method. The PS candidates with large phase noise are eliminated, but point $\mathrm{F}$ is selected (Fig 3e). The actual object of the $\mathrm{F}$ point is the landmark building " BundBull" in the Bund Jinniu square (see Fig 5). It was confirmed by actual data that this building was built on May 14, 2010. As the experiment using SAR images for the earliest time is 2008 , so point $F$ is not reliable. It shows that phase analysis method to extract the PS point is not entirely effective PS point, also can cause misjudgment of PS point.

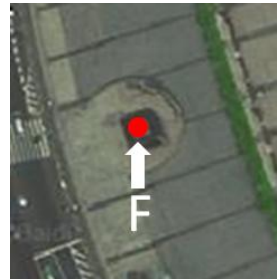

Figure 5. The actual object correspond to $\mathrm{F}$ point

Figure 3f shows the distribution of PS points by our improved method. Firstly, the amplitude threshold method is used to select the points. Since the amplitude threshold method takes into account the high SNR characteristics of pixels, the pixels with weak backscattering properties are removed, such as points $\mathrm{A}$ and $\mathrm{E}$. Then, the dispersion of intensity method was used to select points, and it considers the change of the time series intensity, and eliminated the unstable points, such as points B, $\mathrm{C}, \mathrm{D}$ and $\mathrm{F}$. According to the phase analysis, the PS points with higher phase noise are eliminated, and the high quality PS points are obtained, as shown in Figure 3f. The distribution density of PS points obtained by this method is about 969 points per square kilometer, most of which are distributed in roofs of dykes, roads and the corner and roof of the buildings, and meet the requirements of PSInSAR technology in urban areas. Compared with other methods, the improved method of PS points can not only meet the requirements of reliability and stability, also to eliminate the candidate PS points with large phase noise, and then improve the accuracy of the wrapped phase solution.

3.2.2 Distribution Rationality Analysis: Besides the accuracy and stability, the rationality of PS point distribution is also an important condition to evaluate the quality of PS point. Tongji university medical college building is located on the Siping road campus of Tongji university, where stable angular structures and the concrete surface material produce efficient reflectors that dominate background scattering (Fig 6), electromagnetic waves can be reflected well. Therefore, taking this building as an example, the distribution characteristics of PS points obtained by the improved method are analyzed.

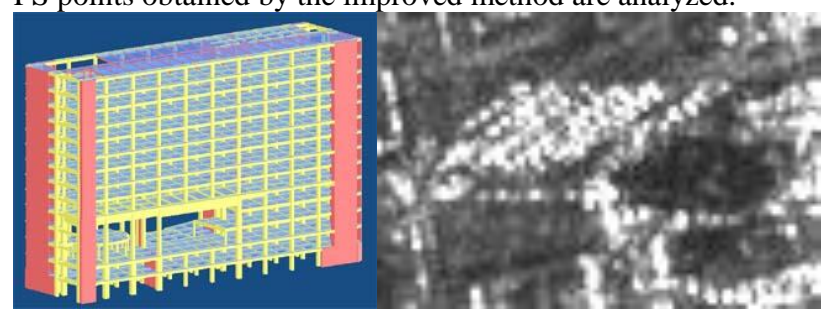

Figure 6. The 3D image and SAR image of Tongji Medical College

(Satellite image is from Baidu Baike, SAR image is COSMOSkyMed descending image.)

Using the improved method proposed in Section 1.4, PS points are extracted for this building. The number of PS points is 91, and the distribution is shown in Figure 7(a):

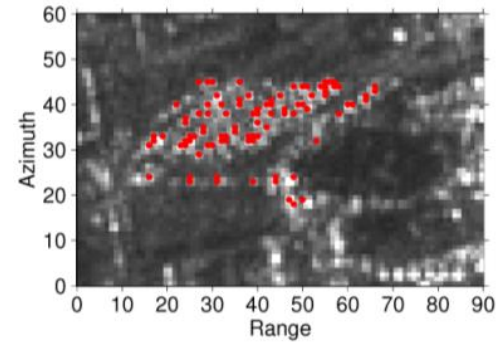

a

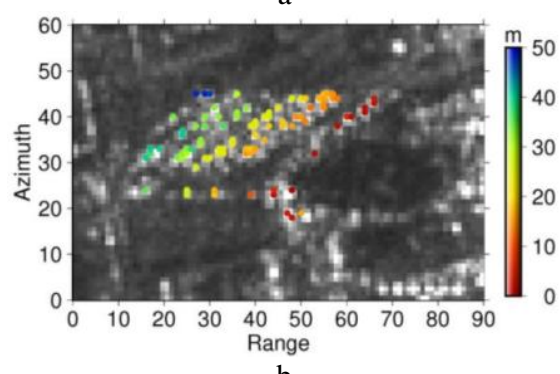

b

Figure 7. PS points distribution and height of Tongji Medical College Building

(a. Distribution of PS points, b. Elevation of PS points) 
In Figure 7(a), the red dots presents PS points, and the distribution of PS points is roughly along the structural shape of the building. After elevation estimation, the height of PS points in this building are obtained, as shown in Figure 7(b). After geocoding, the general structure of the building can be restored approximately, as shown in Figure 8.
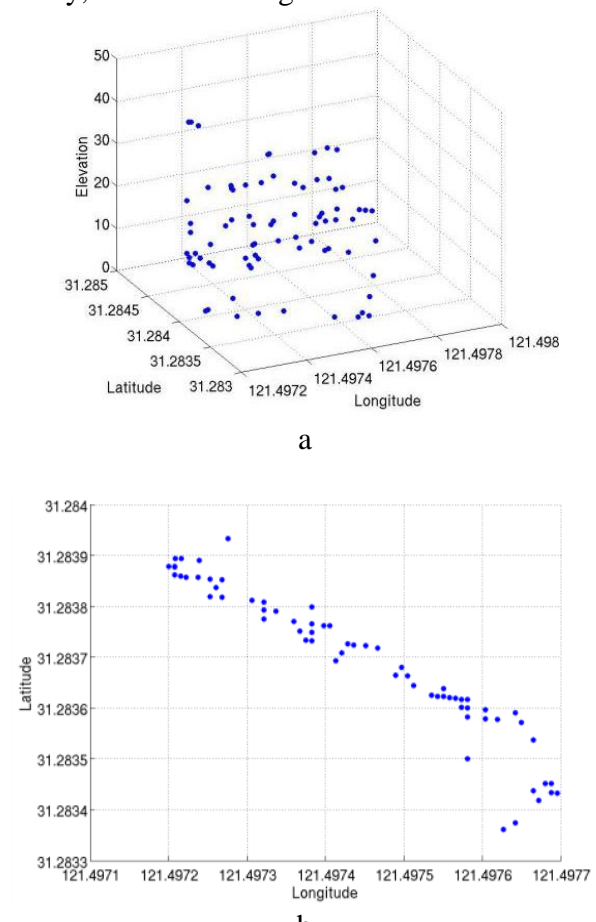

Figure 8. 3D model (a) and horizontal position (b) of PS points on the Tongji Medical College building

It can be seen that the improved PS extraction method can not only meet the requirements of accuracy and stability, but also meet the requirements of the distribution rationality of PS points.

\subsection{The LOS Deformation rate obtained}

According to the improved PS extraction method, the PS points were selected, and the IPTA method in GAMMA software was used to process the full SAR image data. After data process, the deformation result along the line-of-sight of the experimental area is obtained.

3.3.1 The selection of the PS candidate: Firstly, the PS point was detected by the amplitude threshold method, and 1801285 PS candidates were extracted. According to the dispersion of intensity method, the PS candidate points were selected in a rough way, and 277609 PS candidates were selected. The two subsets of candidate points were intersected, and then the interference phase spatial correlation analysis was used to select in the PS candidate points. Finally, 128,075 PS points are obtained, and the point density is about $1,179 / \mathrm{km}^{2}$. The result shows that the selection of PS points is highly correlated with the artificial infrastructure. The PS points are more dense in residential areas and roads, display clear edges of buildings, roads and waters, and clearly linear distribution along the road and dyke.

To verify the superiority of the improved method, the common and effective dual threshold method (Wu et al.,2015) is adopted, and compare it result with our improved method.
The coherence statistics of the PS points selected by the dual threshold method and the improvement method are shown in Figure 9. Among them, the PS points coherence of the improved method are relatively high, concentrated in the 0.85 0.98 . In addition, the high coherent point frequency is higher and the proportion is larger. Whereas, the PS points coherence extracted by the dual threshold method are relatively low.

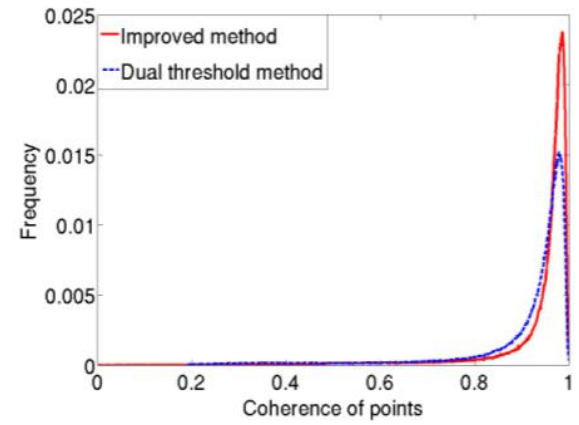

Figure 9. Coherence histogram of PS points

In order to verify the stability of our selected PS points, three feature points (see Fig 10, points A, B, C) are selected in the interest area, and the stability analysis of PS point is analyzed by phase stability analysis. As shown in Table 2, the residual phase mean and the phase standard deviation of the feature points are relatively small, and the stability of PS points is verified.

\begin{tabular}{ccc}
\hline Point & $\begin{array}{c}\text { Residual phase mean } \\
\text { ( rad })\end{array}$ & $\begin{array}{c}\text { Residual phase } \\
\text { standard deviation } \\
\text { (rad) }\end{array}$ \\
\hline A & 0.043 & 0.049 \\
B & -0.029 & 0.0346 \\
C & 0.012 & 0.0121 \\
\hline
\end{tabular}

Table 2. Residual phase means and standard deviations of feature points

The data processing with PSI technology: After determining the two groups of PS points, residual phase can be respectively obtained by subtracting the low-frequency spatial part from the initial differential interference phase. Residual phase mainly includes the nonlinear deformation phase, atmospheric phase and noise (Umberto et al.,1995). First, the Delaunay TIN is established between PS points, and the phase difference can be unwrapped in the temporal domain. When the unwrapped phase in temporal domain is finished, the cost function can be constructed for each phase difference, and the Minimum Cost Flow (MCF) method is used to unwrap (Liao et al.,2003). The nonlinear deformation spatial correlation is relatively small and has low frequency characteristics in temporal domain. The atmosphere delay phase is spatial correlated, but is not correlated in temporal domain and has high frequency in time. Therefore the nonlinear deformation and atmospheric delay phase can be separated by the temporal filtering method and the spatial filtering method, and then the deformation phase is recovered. Finally, the time series of deformation and average subsidence rate in 2008-2010 in interested area are obtained (Fig 10). 


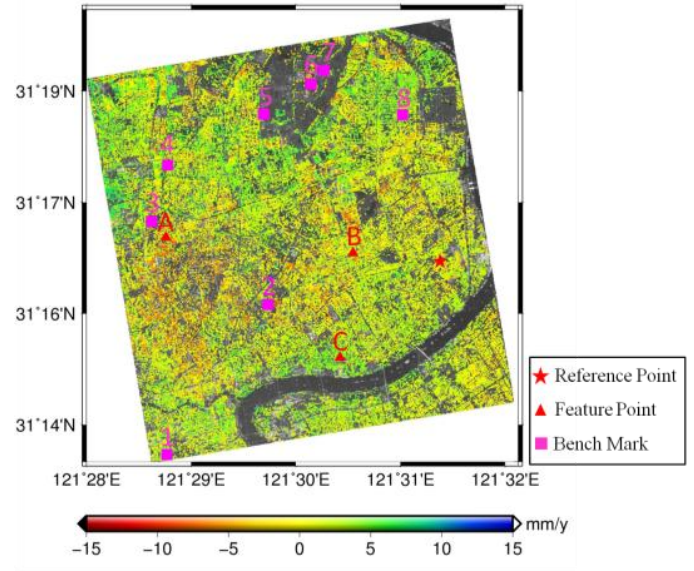

$\mathrm{a}$

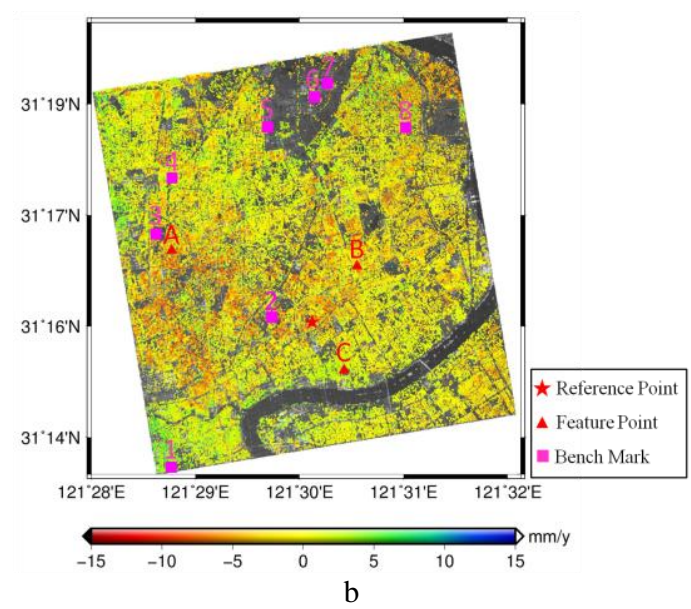

Figure 10. Average subsidence rate of interested area

(a. Deformation rate obtained by the Improved PS detection method. b. Deformation rate obtained by the Dual Threshold method)

Figure 10 shows the average subsidence rate distribution of PS points extracted by the improved method and the dual threshold method respectively. The red star represents the reference point In Figure 10, the ground subsidence rate of the interested area between 2008 and 2010 varies from $-14.6 \mathrm{~mm} /$ year to $4.9 \mathrm{~mm} /$ year. There is an obvious settlement zone: from west to east through the original Zhabei district, through Hongkou district, Yangpu district and extended to the Gongqing National Forest park. There is a sedimentation funnel in the Hongkou district, and the funnel center is the junction of subway line 8 and line 10, adjacent to Siping road subway station. The average subsidence rate of this area is $13.7 \mathrm{~mm} /$ year.

\subsection{Accuracy Assessment}

3.4.1 Internal accuracy: The accuracy of the deformation rate along line-of-sight direction by PSInSAR can be measured by the root-mean-square error (RMSE) of the deformation rate. See Figure 11, the frequency of the RMSE greater than $1 \mathrm{~mm} / \mathrm{y}$ in the deformation rate which obtained by the improved PS selection method is almost 0 , and the overall RMSE is between $0.1 \mathrm{~mm} / \mathrm{y}$ and $0.6 \mathrm{~mm} / \mathrm{y}$. The frequency of the RMSE greater than $1 \mathrm{~mm} / \mathrm{y}$ in the deformation rate obtained by the dual threshold method is greater than 0.05, the RMSE ranges from $0.4 \mathrm{~mm} / \mathrm{y}$ to $1 \mathrm{~mm} / \mathrm{y}$, which is larger, indicating that the accuracy is lower than that of the improved method.

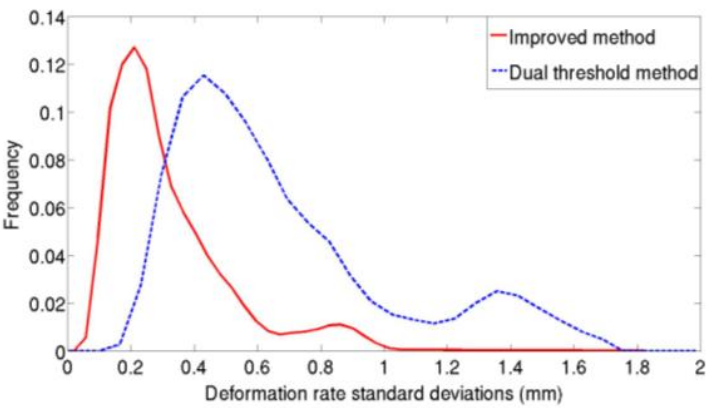

Figure 11. Deformation rate standard deviations

3.4.2 External accuracy: The traditional method of ground subsidence monitoring is the spirit leveling measurement, the accuracy of the spirt leveling is relatively high, and the stability of the measurement data is better. In order to verify the accuracy of PSInSAR monitoring results, we select 8 spirit level monitoring points in the experimental area from 2007 to 2011 (as shown in the pink square points in Fig 10), and compare the results with the leveling results. In order to compare two results, the spirit level observation can be projected to radar line-ofsight direction according to the geometry of SAR image, and then do a comparative analysis. The leveling point expresses the settlement information at a point. While PSInSAR result is a pixel-based deformation information, and a pixel corresponds to a small area in the ground suface. So the two results cannot be directly matched. Therefore, average subsidence rate of the PS points within $200 \mathrm{~m}$ to the levelling point is calculated, and then compared with the leveling results. The comparison results are shown in Figure 12.

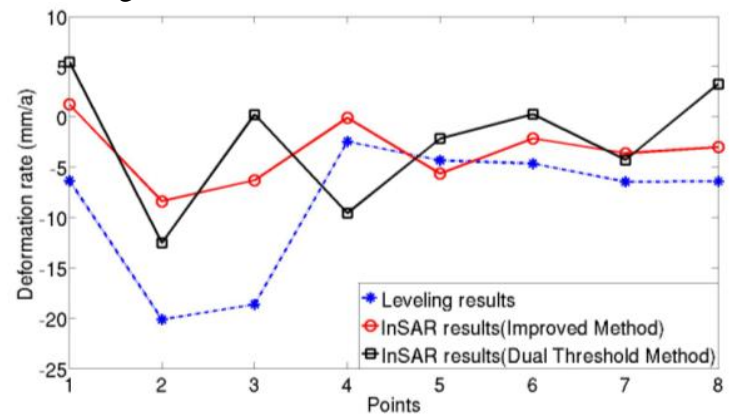

Figure 12. Comparison between PSInSAR results and leveling measurement

The PSInSAR results obtained by the improved PS method are basically consistent with the deformation trend of the level observations (Fig 12). However, the results obtained by the double threshold method are relatively different. It can that the improved PS point detection method is more consistent with the leveling. Because of the observation time is not same (leveling observation time is from 2007 to 2011), the results between levelling and PSInSAR cannot be identical. But look from whole subsidence trend, the results of PSInSAR monitoring are basically consistent with the spirit leveling observation, and indicates that the PSInSAR monitoring results are reliable.

\section{CONCLUSIONS}

(1) Using the improved method to extract high quality PS points Firstly, PS candidates are obtained by the amplitude threshold method and dispersion of intensity method, and then the intersection of the two PS candidates are taken for interferometric phase space correlation analysis. Finally, PS points which meet the requirements of high reflection scattering 
intensity and stability are extracted. After experimental verification, the improved method is effective and reliable.

(2) Experiment adopted high resolution SAR images which acquired in periods of 2008-2010, the sedimentation rate distribution map is obtained, and compared with the spirit leveling data, the results of improved method are proved to be stable and reliable. The result of the experiment find a settlement funnel area at the junction of Yangpu and Hongkou district, with the maximum settlement rate of $14.6 \mathrm{~mm} /$ year.

(3) In urban areas, there is a high density of PS points. PSInSAR technology can be used to monitor ground subsidence more widely and accurately. Compared with spirit leveling measurement, PSInSAR technology has higher spatial resolution, and the ground settlement information obtained is more specific and intuitive.

\section{ACKNOWLEDGEMENTS}

This research was supported by the State Key Development Program for Basic Research of China (No. 2013CB733304), the DAAD Thematic Network Project(ID:57173947) and the National Science Foundation (No. 41674003). We thank the Beijing Vastitude Technology Co., Ltd. for providing the COSMO-SkyMed images.

\section{REFERENCES}

Hooper, A. J., 2006. Persistent scatter radar interferometry for crustal deformation studies and modeling of volcanic deformation.

Chen Q, Liu G, Li Y, et al., 2006. Automated detection of permanent scatterers in Radar interferometry: algorithm and testing results. Acta Geodaetica et Cartographica Sinica, 2, pp.112-117. (in Chinese)

Fan, Jiao , Gao, et al., 2016. Comparison research of high coherent target selection based on InSAR time series analysis. Journal of Geo-information Science,18(6), pp.805-814. (in Chinese)

Ferretti, A., Fumagalli, A., Novali, F., Prati, C., Rocca, F., \& Rucci, A., 2011. A new algorithm for processing interferometric data-stacks: SqueeSAR. IEEE Transactions on Geoscience and Remote Sensing, 49(9), pp.3460-3470.

Ferretti, A., Prati, C., \& Rocca, F., 2001. Permanent scatterers in SAR interferometry. IEEE Transactions on geoscience and remote sensing, 39(1), pp.8-20.

Gao S., 2015. A method of combining PS and DS for InSAR time series analysis. Beijing: Peking University. (in Chinese)

Hooper, A., 2007. A combined multi-temporal InSAR method incorporating persistent scatterer and small baseline approaches. In ESA Fringe 2007 Workshop, Frascati, Italy.

Hooper, A., 2008. A multi-temporal InSAR method incorporating both persistent scatterer and small baseline approaches. Geophysical Research Letters, 35(16).

Hooper, A., Segall, P., \& Zebker, H., 2007. Persistent scatterer interferometric synthetic aperture radar for crustal deformation analysis, with application to Volcán Alcedo, Galápagos. Journal of Geophysical Research: Solid Earth, 112(B7).
Hooper, A., Zebker, H., Segall, P., \& Kampes, B., 2004. A new method for measuring deformation on volcanoes and other natural terrains using InSAR persistent scatterers. Geophysical research letters, 31(23).

Liu G, Chen Q, Luo X, et al.,2009. Theory And Method Of Permanent Scatterer Radar Interference. Beijing: Science Press. (in Chinese)

Liao M, Lin H.,2003. Radar Interferometry - Principle And Signal Processing Base. Beijing: The Mapping Publishing Company . (in Chinese)

Liao M, Pei Y, Wang H, et al.,2012. Monitoring Of Ground Subsidence In Shanghai. Shanghai Land and Source, 33(3), pp.5-10. (in Chinese)

Nie Y, Liu G, Shi J., 2012. Application Of Psi Technology In Surface Deformation Monitoring. Science of Surveying and Mapping,38(2), pp.80-83.(in Chinese)

Parizzi, A., Perissin, D., Prati, C., \& Rocca, F., 2005. Monitoring Tianjin subsidence with the Permanent Scatterers technique. In Proc. of Dragon Symposium 2005, pp. 1-6.

S. Gernhardt , N. Adam , M. Eineder \& R. Bamler, 2010. Potential of very high resolution SAR for persistent scatterer interferometry in urban areas, Annals of GIS, 16:2, pp.103-111.

Spagnolini, U., 1995. 2-D phase unwrapping and instantaneous frequency estimation. IEEE Transactions on Geoscience and Remote Sensing, 33(3), 579-589.

Wu, J., \& Hu, F., 2015, September. Ground subsidence monitoring of high speed roads by using PS-InSAR method. In Synthetic Aperture Radar (APSAR), 2015 IEEE 5th AsiaPacific Conference, pp. 853-858. IEEE.

Wu, J., Hu, F., \& Yang, T., 2016, July. Ground subsidece monitoring along the levee of Huangpu River by multi-platform high-resolution SAR images. In Earth Observation and Remote Sensing Applications (EORSA), 2016 4th International Workshop, pp. 227-231. IEEE.

Zhang H, Wang C, Wu T, et al., 2009. Research On Dinsar Method Based On Coherent Target. Beijing: Science Press. (in Chinese)

Zhao, J., \& Wu, J., 2014, June. Ground subsidence monitoring in western region of Shanghai Maglev by Cosmo-SkyMed ascending and descending SAR images. In Earth Observation and Remote Sensing Applications (EORSA), 2014 3rd International Workshop, pp. 28-32. IEEE. 